open access

\section{EDITORES}

- Miguel Oliveira Jr. (UFAL)

- René Almeida (UFS)

\section{AVALIADORES}

- Adan Phelipe Cunha (USP)

- Adriana Tavares (UFRRJ)

\section{SOBRE OS AUTORES}

- Adriana Maria Tenuta de Azevedo Conceptualização, Metodologia, Administração do Projeto,

Visualização, Escrita-Rascunho Original, Escrita, Análise e Edição.

- Stefânla Aparecida de Lima Silva Conceptualização, Análise Formal, Visualização, Escrita - Rascunho Original, Escrita, Análise e Edição.

DATAS

- Recebido: 20/08/2021

- Aceito: 13/09/2021

- Publicado: 18/11/2021

\section{COMO CITAR}

TENUTA, A. M.; SILVA, S. A. L. (2021). O particípio presente como pré e pósmodificador do sintagma nominal: uma análise comparativa da produção de aprendizes brasileiros e de falantes nativos do inglês. Cadernos de Linguística, v. 2, n. 4, e574.
RELATO DE PESQUISA

\section{O PARTICÍPIO PRESENTE COMO PRÉ E PÓS-} MODIFICADOR DO SINTAGMA NOMINAL: UMA ANÁLISE COMPARATIVA DA PRODUÇÃO DE APRENDIZES BRASILEIROS E DE FALANTES NATIVOS DO INGLÊS

\author{
Adriana Maria Tenuta de AZEVEDO (iD $\boldsymbol{\Sigma}$ \\ Universidade Federal de Minas Gerais (UFMG) \\ Stefânia Aparecida de Lima SILVA (D) $\boldsymbol{X}$ \\ Universidade Federal de Minas Gerais (UFMG)
}

\section{RESUMO}

Este artigo investigou comparativamente a produção escrita de aprendizes brasileiros e de falantes nativos de inglês quanto ao uso de pré e de pós-modificadores do sintagma nominal realizados pelo particípio presente -ing. Objetivou-se um levantamento quantitativo das ocorrências desses elementos no corpus de aprendizes CorlsFInglês e no corpus de falantes nativos LOCNESS, ambos compostos por textos acadêmicos. Para o tratamento dos dados, foi utilizado o software AntConc. Foram encontradas mais ocorrências de -ing modificadores, tanto pré quanto pós, pelos falantes nativos em relação aos aprendizes. O nível de proficiência linguística dos aprendizes (de A2 a B2 do QECRL') não sendo condizente com a produção de sintagmas nominais mais complexos pode explicar essa parte dos resultados. Considerando-se as gramáticas de L1 e L2 dos aprendizes, o particípio presente (-ndo e -ing, respectivamente) desempenha a função sintática de pós-modificador de sintagma 
nominal em ambas. Porém, essa forma não finita do verbo ocorre como pré-modificador apenas no inglês. A outra parte dos resultados revela que os falantes nativos empregaram a forma -ing como pósmodificador cerca de proporcionalmente 2 vezes mais do que os aprendizes brasileiros. No caso da pré-modificação, verificou-se que os falantes nativos utilizaram 4 vezes mais itens marcados por -ing do que os aprendizes. Essas diferenças numéricas encontradas nos dados foram analisadas como influência do português na produção dos textos em inglês pelos aprendizes brasileiros, o que pode estar relacionado a uma estratégia de aprendizagem.

\section{ABSTRACT}

This article investigated the written production of Brazilian learners of the English language, comparatively to native speakers, regarding the use of -ing present participles as pre and post-modifiers in noun phrases. We aimed at quantifying the occurrences of these elements in the CorlsFInglês, a learners' corpus, as well as in LOCNESS, a native speakers' corpus, both composed of academic texts. For data processing, the AntConc software was used. We found more occurrences of the non-finite form of the verb both as pre and as post-modifiers produced by native speakers in relation to learners. The learners' linguistic proficiency level (from A2 to B2 of CEFLR) being inconsistent with the production of rather complex noun phrases may explain this part of the results. Taking into account the grammar systems of the learners' L1 and L2, the present participle (-ndo and -ing, respectively) has the syntactic function of postmodifier in the noun phrase in both systems. However, this non-finite form of the verb occurs as pre-modifier only in English. The other part of the results revealed that the native speakers used the -ing as post-modifier proportionally about twice as much as the Brazilian learners. As for premodifiers, the data indicated that the native speakers used -ing items four times more than the learners did. These numerical differences were analyzed as influence from Portuguese in the English text production of Brazilian learners, what can be related to a learning strategy.

\section{PALAVRAS-CHAVE}

Pré-modificador de sintagma nominal; pós-modificador de sintagma nominal; particípio presente; interlíngua; estratégia de aprendizagem.

\section{KEYWORDS}

Pre-modifier in a noun phrase; post-modifier in a noun phrase; present participle; past participle; interlanguage; learning strategy. 


\section{INTRODUÇÃO}

A investigação da produção de aprendizes de uma língua estrangeira, por si só ou em comparação com a produção de falantes nativos dessa língua, pode lançar luz sobre aspectos da estruturação linguística que são de difícil compreensão em um contexto de aprendizagem. Nesse sentido, a produção numericamente reduzida de um conteúdo gramatical por parte do aprendiz em comparação com uma expectativa mais volumosa de ocorrências, ou a produção desse conteúdo gramatical com características marcadamente distintas da produção do falante nativo, são sinais de uma defasagem na aprendizagem, bem como de que a interlíngua do aprendiz exibe padrões de uso específicos.

Este artigo teve como objetivo abordar o uso do particípio presente (-ing) da língua inglesa, que é uma forma não finita do verbo, nas funções sintáticas de pré e de pósmodificador do sintagma nominal, em textos produzidos por aprendizes brasileiros de inglês, dando enfoque à análise quantitativa dessa produção. Houve, neste trabalho, o apoio metodológico na linguística de corpus, uma vez que os dados analisados foram retirados de dois corpora. O primeiro corpus utilizado foi o CorlsF-Inglês, Corpus do Inglês Sem Fronteiras ${ }^{2}$, que é composto de 1646 textos acadêmicos dos gêneros carta, carta de intenção, e-mail, resumo e ensaios narrativos, descritivos e argumentativos, tendo o número total de 278684 palavras. Os textos do CorlsF-Inglês foram produzidos por aprendizes brasileiros da língua inglesa, universitários de níveis entre A2 e B2 do Quadro Comum Europeu de Referência Linguística. O corpus utilizado como referência foi o LOCNESS, Louvain Corpus of Native English Essays ${ }^{3}$, composto por ensaios literários e argumentativos produzidos por estudantes universitários da língua inglesa, contendo 324.304 palavras, originárias de 322 textos.

Os textos dos corpora analisados, apesar de serem de gêneros variados, são todos da esfera acadêmica, o que situa o trabalho na perspectiva do letramento acadêmico. Optamos por trabalhar com essa variedade para obtermos um panorama geral da produção de -ing pré e pós-modificador nesse contexto amplo. Estudos futuros deverão ser realizados com o enfoque específico em um gênero apenas, como, por exemplo o ensaio argumentativo, que é o mais representativo em ambos os corpora.

O sintagma nominal da língua inglesa é composto essencialmente por um núcleo nominal, prototipicamente realizado por um substantivo, ou um pronome, e, por vezes, por um elemento verbal não-finito. Esse núcleo pode ser circundado por elementos tais como,

2 Este corpus foi construído de 2014 a 2016, com textos produzidos por estudantes universitários da UFLA, UFMG UFPI e UFSJ

3 Este corpus é originário da Bélgica, compilado pela professora Silviane Granger. Seus colaboradores são estudantes nativos da língua inglesa e de instituições como as Universidades da Carolina do Sul e Michigan, USA, e de Surrey, UK. 
o determinante e o pré-modificador, em posição anterior ao núcleo, e o pós-modificador, em posição posterior a ele. Para Downing e Locke (2006) e Downing (2015), os prémodificadores têm a função de classificar ou descrever o núcleo do sintagma nominal, enquanto os pós-modificadores acrescentam informação sobre esse núcleo, identificandoo ou definindo-o.

Os determinantes possuem uma gama de possibilidades de realização e essa possibilidade de realização variada também acontece em relação aos pré e aos pósmodificadores. Como pré-modificadores, encontram-se adjetivos, substantivos, o particípio presente (-ing) e o particípio passado (-en).

(1) a) adjetivo:

one fine morning in October 1969. (DOWNING, 2015, p. 362)

b) substantivo:

a brick wall. (HUDDLESTON; PULLUM, 2005, p. 95)

c) particípio presente:

a sleeping child. (HUDDLESTON; PULLUM, 2005, p. 95)

d) particípio passado:

an experienced woman worker. (BIBER et al., 1999, p. 599)

Há uma diferença significativa quando se comparam as possibilidades de realização desses pré-modificadores da língua inglesa com aqueles da língua portuguesa, língua materna do aprendiz de inglês em nossa pesquisa. No português, na função sintática de pré-modificador do sintagma nominal, não há a possibilidade de ocorrência do substantivo, nem das formas não finitas do verbo, como o particípio presente, o gerúndio ou o particípio passado. "O núcleo de um sintagma nominal admite a presença de determinantes (DET) [...] e de modificadores (MOD), que, no caso, são os adjetivos, ou expressões adjetivas." (CUNHA; CINTRA, 2017, p. 137). Assim como descrito em Cunha e Cintra, os pré e os pósmodificadores do sintagma nominal do português, no geral, não são apresentados pelas gramáticas como duas categorias distintas e os elementos que integrariam cada uma dessas categorias aparecem, nas gramáticas, de forma pulverizada, ou seja, não especificamente agrupados.

Encontramos, em Perini (2005), os adjetivos ou sintagmas adjetivais entre os elementos pré-nucleares (que incluem, além do que estamos tratando por pré-modificadores, todos os determinantes). São exemplos desses adjetivos os itens sublinhados em (2):

(2) a) Cada inesquecivel viagem

b) Todo bom professor (PERINI, 2005, p. 100) 
Com relação à realização do elemento pós-núcleo do sintagma nominal, as línguas inglesa e portuguesa exibem possibilidades muito similares, sendo que a grande diferença se deve ao uso privilegiado do adjetivo na língua portuguesa, o que não ocorre na língua inglesa. No entanto, como já revelado, em gramáticas do português, muitas vezes, nem há referência a pré e a pós-modificadores separadamente. Sabemos, porém, que, em ambas as gramáticas, encontram-se, na posição sintática pós-núcleo, além de sintagmas preposicionados, o particípio passado e o particípio presente, sozinhos ou em orações subordinadas adjetivas/relativas expandidas ou reduzidas.

Para compormos um quadro das possiblidades de pós-modificação de sintagma nominal no português, juntamos exemplos de autores variados:

(3) a) adjetivo ou sintagma adjetival:

A criancinha doente melhorou. (OTHERO, 2008, p. 69);

O homem orgulhoso de seus feitos descansa em paz. (OTHERO, 2008, p. 122)

b) sintagma preposicionado:

O menino da camisa verde a caba de chegar. (OTHERO, 2008, p. 70);

As folhas das árvores começaram a cair. (OTHERO, 2008, p. 89)

c) complemento oracional:

Ele tem medo de que ela chore. (OTHERO, 2008, p. 111);

Ele tem medo que ela chore. (OTHERO, 2008, p. 111)

d) oração subordinada adjetiva restritiva:

És um dos raros homens que têm o mundo nas mãos. (CUNHA, CINTRA, 2017, p. 618)

e) oração subordinada adjetiva explicativa:

O homem, que se considera racional, muitas vezes age animalescamente. (CIPRO, INFANTE, 2008, p. 427)

f) oração reduzida de infinitivo:

A morte é o último inimigo a ser destruide por Cristo. (CEGALLA, 2008, p. 411)

g) oração reduzida de particípio passado:

Esta é a notícia divulgada pela imprensa. (que foi divulgada pela imprensa) (CEGALLA, 2008, p. 413)

h) oração reduzida de gerúndio: 
Havia ali crianças pedindo esmola. (CEGALLA, 2008, p. 412). Observamos que este uso se trata do particípio presente.

São exemplos, também de vários autores, de possíveis realizações de pós-modificador de sintagma nominal na língua inglesa:

(4) a) sintagma preposicionado:

a new album by a top musician. (DOWNING, 2015, p. 401)

b) oração relativa finita:

the man who is standing in the corridor. (DOWNING, 2015, p. 401); the email $L$ sent this morning (HUDDLESTON; PULLUM, 2005, p. 96)

c) oração relativa não-finita:

the man standing in the corridor (-ing cl.); the man to consult is Jones (to-inf. cl.) (DOWNING, 2015, p. 401); a letter written by his uncle (-en cl.). (HUDDLESTON; PULLUM, 2005, p. 96)

d) adjetivo ou sintagma adjetivo:

the best hotel available; a room full of furniture. (DOWNING, 2015, p. 401)

e) advérbio:

the flat upstairs. (DOWNING, 2015, p. 401)

f) complemento nominal:

The issue that there is global warming is debatable. (DOWNING, 2015, p. 401)

g) sintagma nominal (aposto):

the opera 'Carmen'; our friend the mayor. (HUDDLESTON; PULLUM, 2005, p. 96)

h) sintagma nominal (não aposto):

a woman my age; a rug this colour. (HUDDLESTON; PULLUM, 2005, p. 96)

Segundo Biber et al (1999, p. 604), a pós-modificação mais comumente encontrada em textos acadêmicos de falantes nativos do inglês é a realizada por sintagma preposicionado, em uma média de $80 \%$ dos registros de pós-modificação. Isso nos mostra que a pós-modificação no inglês não ocorre preferencialmente por meio das formas não finitas do verbo. 
A influência da L1 nas produções de aprendizes no processo de aquisição da L2 é reconhecida desde as práticas da análise contrastiva, que, de acordo com Durão (2005, p. 8-9), é uma teoria que busca identificar semelhanças e diferenças entre as línguas. $\mathrm{Na}$ literatura de ASL (Aquisição de Segunda Língua), os conceitos de transferência e interferência relacionados à aprendizagem de uma L2 (GASS; SELINKER, 1992), em qualquer nível da estruturação linguística, semântico, sintático, fonológico ou outro, reforçam a ideia de que os aprendizes utilizam seu conhecimento da L1 para aquisição de padrões da língua-alvo. Selinker (1972) relaciona a noção de transferência com a de interlíngua, que seria a gramática mental dos aprendizes, uma variante especial entre a língua-alvo e a materna. Para Ellis (1995), a interlíngua constitui um sistema flexível, dependente do repositório linguístico do aprendiz relacionado a $L 1$ e a $L 2$, formado por fatores que podem tanto ser externos quanto internos à língua.

Gass e Selinker (1992) juntaram em sua coletânea trabalhos clássicos relacionados ao tema da transferência linguística e incluíram novos estudos que mostravam que o conceito teórico ainda vigorava. Schachter (1992), um desses trabalhos, aborda o processo de transferência linguística como um fenômeno involuntário. A autora enfoca adultos aprendizes de L2 e argumenta que esses indivíduos formulam teses e hipóteses sobre o funcionamento dessa língua, envolvendo transferência da L1. Marvin Levine (1975, apud SCHACHTER, 1992) descreve esse fenômeno como hypothesis theory ou simplesmente $H$. Theory. Tomando por base essa teoria da hipótese, Schachter conclui que o processo de transferência envolve a busca de solução para problemas na língua-alvo, no conhecimento prévio do aprendiz. Corder (1992), em outro artigo da coletânea de Gass e Selinker (1992), como uma voz parcialmente discordante, nega a possibilidade de interferência, no sentido de que o conhecimento de L1 possa ser prejudicial de alguma forma ao aprendizado de L2. O autor sustenta que, quando se usam estruturas de L1 em L2, está ocorrendo o emprego de uma estratégia de aprendizagem. Isso só acontece relacionado a aspectos nos quais L1 é similar a L2. Para Corder, então, é obsoleta a noção de interferência, mas não necessariamente de transferência. Kroll e Stewart (1994) entendem que, à medida que a proficiência na língua-alvo aumenta, há um processo gradual no sentido de se adquirir independência de interferências. Mais recentemente, o conceito de transferência é apontado por Alvarez (2002) como indiscutível; essa autora avalia que a "tarefa é descobrir quando e por que se produz a transferência". Trabalhos mais atuais, como Viñas-guasch, Gathercole e Stadthagen-gonzalez (2017); Fricke et al. (2019), continuam reconhecendo a relação de transferência entre L1e L2.

Com base nas possibilidades de realização dos elementos estruturantes dos sintagmas nominais da língua materna (L1) em comparação com aqueles da língua-alvo (L2) do 
aprendiz, é plausível considerar a hipótese da ocorrência de uma influência ${ }^{4}$ do português sobre a produção de brasileiros falantes da língua inglesa. Estamos considerando influência como um termo abrangente com um sentido positivo de 'motivação' ou 'estímulo' para o uso ou a automatização de algum padrão da L1 também ocorrente na $L 2$, não na perspectiva de interferência ou transferência em que se têm padrões sintáticos exclusivos da L1 sendo usados na L2.

Em nossa investigação, consideramos, então, a hipótese de que haja proporcionalmente mais ocorrências de pós-modificadores com -ing do que ocorrências de pré-modificadores com essa terminação, uma vez que na língua portuguesa são encontradas estruturas reduzidas de particípio na posição pós-núcleo do sintagma nominal, espelhando a gramática da língua inglesa. Como pré-modificador, não se esperaria grande ocorrência de -ing, uma vez que a L1 dos aprendizes distingue-se da L2, não prevendo a utilização da forma verbal não finita -ndo na referida posição sintática. Todo esse quadro constitui uma situação propícia para a referida influência da L1 na produção de L2. Para ilustrar nossa hipótese, entendemos que um aprendiz da língua inglesa falante do português produziria, mais naturalmente, the election coming do que the coming election, justamente por transferência da L1.

\begin{tabular}{l|c|c|l|l} 
& \multicolumn{2}{|l}{} \\
\hline $\begin{array}{c}\text { Padrão esperado de } \\
\text { produção na língua } \\
\text { inglesa }\end{array}$ & determinante & pré-modificador & núcleo & pós-modificador \\
\hline Falante Nativo & The & coming & election & - \\
\hline Aprendiz & The & - & election & coming
\end{tabular}

Quadro1. Possível preferência para a formação de sintagma nominal com modificador -ing.

O aprendiz vai escolher modificar o substantivo usando um -ing pós-modificador ao invés de um -ing pré-modificador devido às referências gramaticais de sua L1. Para esse aprendiz ter condição de apreender e categorizar a forma -ing como elemento prémodificador e se tornar capaz de produzir essa estrutura, seria necessário um tempo maior de exposição a inputs da L2, ou uma instrução gramatical específica.

Assim, em uma perspectiva pedagógica, considera-se importante uma atenção especial ao elemento linguístico que seja distinto nas gramáticas de L1 e de L2, para que os aprendizes cheguem a atingir um nível desejável de fluência, vindo a produzir estruturas percebidas como mais naturais para um falante nativo, aos moldes, por exemplo, da

4 Nossa perspectiva para o termo é aproximada à de seus sentidos dicionarizados: "Poder de influenciar e modificar o pensamento ou o comportamento de outrem sem o uso da força ou da imposição" ou "Efeito modificador produzido por agentes físicos ou da natureza sobre os seres humanos e as coisas” (MICHAELIS, 2021). 
proposta de Consciousness-Raising (C-R) de Rutherford (1987), defendida em Tenuta (1999), que postula:

\begin{abstract}
que o conhecimento da L2 pode ser relacionado e integrado ao conhecimento consciente da L1, como um primeiro passo em direção à automatização do conhecimento da L2. Essa visão procura construir ligações entre o "conhecimento" da L1 e a "ignorância" da L2, sem preocupação excessiva com a interferência da NL [native language]. Rutherford (1987) analisa aspectos característicos da interlíngua de aprendizes da língua inglesa como $L 2$ e enfatiza a relevância de se trabalhar com estes aprendizes para levá-los à conscientização das diferenças existentes entre essa interlíngua e a língua alvo. (TENUTA, 1999, p. 165)
\end{abstract}

O objetivo do processo de ensino/aprendizagem da língua estrangeira deve ser, então, o de conseguir a automatização referente à produção do aprendiz, isto é, o de fazer com que sua interlíngua reflita o conhecimento automatizado da L2. Essa visão de ensino/aprendizagem atende tanto à perspectiva de ASL (Aquisição de Segunda Língua) quanto à da ILF (Inglês como Língua Franca) (RANTA, 2018). Uma vez identificados padrões específicos da língua do aprendiz, por meio de pesquisas que, como esta, baseiam-se em corpora ampliados, é possível a realização de um trabalho que, por um lado, enfoque a investigação de causas geradoras dos padrões e, por outro, vise à proposição de estratégias pedagógicas relacionadas a esses padrões. No caso da presente investigação, a qual envolve a análise numérica da produção de prémodificadores em comparação com a de pós-modificadores na forma -ing, o resultado alcançado revelaria a direção em que deve acontecer um trabalho pedagógico específico.

\title{
1. METODOLOGIA
}

Nossa pesquisa relaciona-se com uma série de estudos que também verificaram as características da produção escrita de aprendizes de inglês nativos e não nativos, usando metodologia de corpus, como Biber et al. (1999), Granger (1997) e Costa (2017). Neste trabalho, para a separação das ocorrências sob análise, utilizou-se o software AntConc, o qual possui recursos que possibilitam a geração e a visualização de listas de frequência, bem como de linhas de concordância. Procuraram-se nos corpora, com o auxílio desse software, as sequências -ing.

Foram encontradas 6762 linhas de concordância com ocorrências de -ing no CorlsfInglês e 9182 linhas de concordância com ocorrências de -ing no LOCNESS. Em seguida, essas linhas de concordância foram transferidas para planilhas do Excel, a fim de que fossem permitidas análises mais específicas. A partir desse ponto, foi realizado um trabalho manual com os dados selecionados de ambos os corpora.

Foram imediatamente desprezadas todas as linhas de concordância que continham palavras terminadas em -ing nas quais essa sequência de letras não correspondia ao sufixo formador de particípio presente. Entraram nessa categoria palavras como during, 
something, everything, king, bring etc. Além dessas, foram também retiradas as linhas que continham elementos verbais com o sufixo -ing em formas de particípio presente do aspecto progressivo: (is) going, (are) reading, etc. Foram ainda descartadas da planilha as linhas de concordância cuja estruturação encontrava-se prejudicada a ponto de não se ter certeza da função sintática da forma verbal com -ing, como em: ...80's the movie Back to the future wiriting by Robert Zemeckis and Bob Gale... Por fim, foram computadas na análise as linhas de concordância com palavras terminadas por-ing separadas em duas categorias: aquelas em que o item lexical com terminação -ing realizava a função de pré e as em que esse item exercia a função de pós-modificador de SN.

Considerando-se as linhas de concordância com pré-modificadores, foram retiradas ainda aquelas que continham itens lexicais com sufixo -ing, compreendido ou não como formador de particípio presente, mas que são muito entrincheiradas ${ }^{5}$ como adjetivos: interesting, boring, amazing, outstanding, annoying, outgoing, astounding, hard-working, stunning, disgusting. Esses itens possuem alta frequência de uso na língua inglesa e têm como sinônimos outros itens lexicais adjetivos, com os quais podem ser intercambiados: nice, tedious, wonderful etc. Para qualquer falante, até mesmo para o aprendiz, essas palavras são, de imediato, vistas ou apreendidas como adjetivos e não como formas verbais não finitas. Outros itens terminados em -ing que não foram computados em nossa pesquisa como elementos pré-modificadores não finitos foram swimming, shopping e living, integrantes das expressões swimming-pool, swimming-trunks, shopping mall, shopping center, living room, uma vez que essas expressões constituem substantivos compostos da língua inglesa e as palavras em -ing que integram esses substantivos não são processadas como itens verbais não finitos independentes. ${ }^{6}$

Após esses procedimentos, no CorlsF-Inglês, restaram 154 pré-modificadores de sintagmas nominais na forma -ing, exemplificados pelos itens sublinhados em (5) a), b) e c):

(5) a) I agree and disagree with the following statements.

b) This world of the underlying cause we call reality.

c) ...the importance of recycling and organizing a recycling collection...

Já no LOCNESS, restaram 793 pré-modificadores de sintagmas nominais na forma ing, cujos exemplos são os itens sublinhados das linhas de concordância em (6) a), b) e c):

5 Termo utilizado por Langacker (2008) para se referir a unidades linguísticas já enraizadas, de alta frequência, facilmente acessadas, de baixo custo de processamento cognitivo para sua compreensão.

6 Nossa análise vai ao encontro que Downing (2015: 400), que distingue alguns modificadores participiais como verbal nouns ("como gardening em gardening gloves") e outros que "se tornaram adjetivos e admitem gradação por estarem sempre próximos ao núcleo nominal (como interested, bored, exciting). 
(6) a) In the sporting world, there will always be injuries.

b) arguments which helps to adequately debate their differing views on Dr Kevorkian and his medical practices.

c) The trade unions were powerless to stop the rising unemployment, and were often wrongfooted as they...

Em relação às linhas de concordância com pós-modificadores de SN, como a quase totalidade dessas ocorrências era de estruturas oracionais adjetivas, mostrou-se necessário realizar a correta identificação dessas orações adjetivas, também chamadas de orações relativas reduzidas de particípio presente (separando-as das adverbiais função de adjunto, e das nominais - função de sujeito ou de complemento). Apenas as estruturas adjetivas foram computadas nas planilhas finais.

A planilha do CorlsF-Inglês exibiu 359 pós-modificadores de sintagmas nominais na forma -ing, o que está exemplificado nos itens sublinhados nas linhas de concordância selecionadas a seguir:

(7) a) Even with the religion influence becoming weaker, the religious values...

b) ...Paris has restaurants effering typical and refined dishes.

c) It is well known that children at early stages of development are more likely to understand and build up comparisons between different languages, making the task of learning another vocabulary easier.

No LOCNESS, foram encontrados 907 pós-modificadores de sintagmas nominais na forma -ing, como os que estão nos exemplos abaixo:

(8) a) ... or vise (sic) versa for the men watching women.

b) People living without shelter has been a problem in America...

c) While there are many more issues dealing with adoption...

Após havermos quantificado as ocorrências de -ing como pré e como pósmodificadores de SN, calculamos a média de ocorrência desses elementos por 100.000 palavras nos dois corpora. Nossos resultados foram:

\begin{tabular}{l|c|c} 
& LOCNESS & CorlsF-Inglês \\
Pré-modificador & 244,5 & 55,3 \\
\hline Pós-modificador & 279,7 & 128,8
\end{tabular}

Quadro 2. Ocorrências de modificadores-ing nos corpora por 100.000 palavras. 
De posse desses números, fizemos a comparação entre os dois corpora, em relação à utilização do -ing nas duas funções modificadoras do SN:

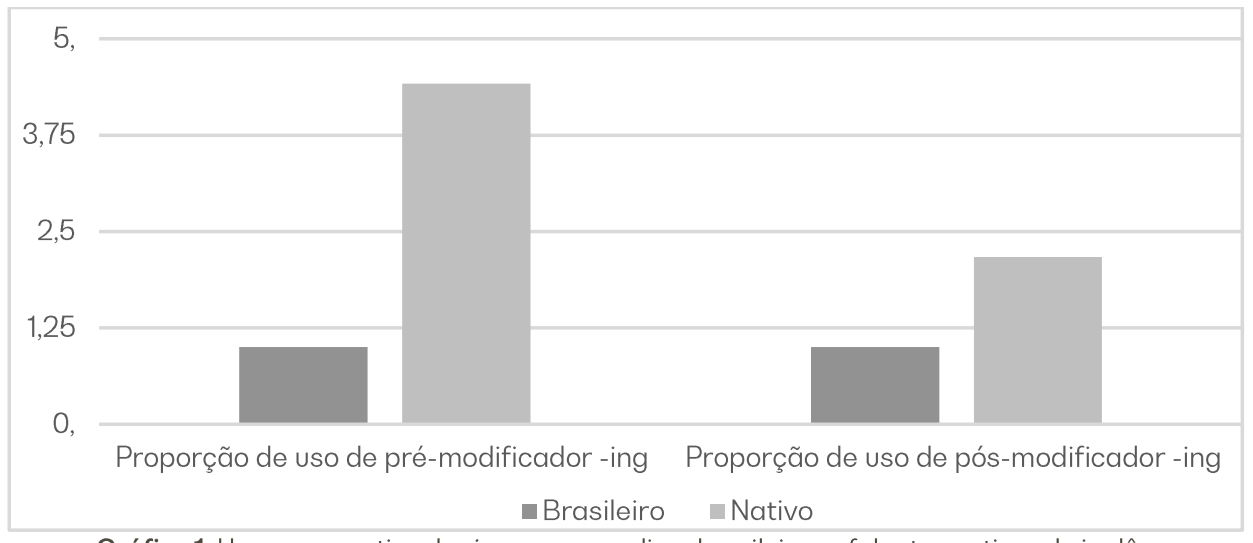

Gráfico 1. Uso comparativo de -ing por aprendizes brasileiros e falantes nativos do inglês.

Apresentamos também, no Gráfico 2, a proporção do uso do -ing nas funções de pré e de pós-modificador por aprendizes brasileiros e falantes nativos. Esse gráfico compara, assim, as proporções, ou seja, a produtividade desse item em cada uma das funções nos dois corpora analisados:

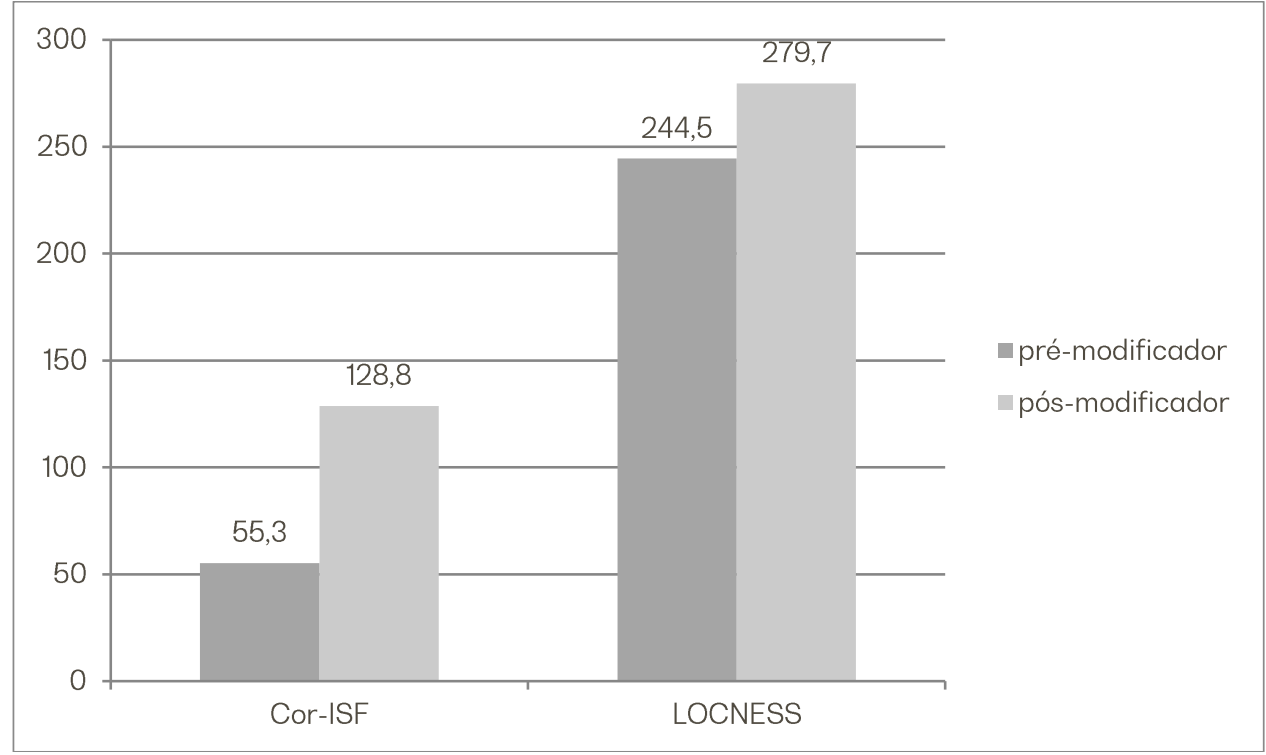

Gráfico 2. Proporção do uso do -ing nas funções de pré e de pós modificador em cada corpus.

Os aprendizes empregam a forma não finita do verbo 2,17 vezes menos na posição de pós-modificador e 4,42 vezes menos na posição de pré-modificador em relação aos falantes nativos. Para o aferimento desse resultado, foi realizado um cálculo de proporção simples, dividindo-se o número de ocorrências em 100.000 palavras de pré e de pósmodificadores com -ing de cada um dos corpora. 
Assim, pode-se esperar que o aprendiz utilize um -ing na função de pós-modificador em apenas $46,04 \%$ das vezes em que um falante nativo o usaria. Em relação à pré-modificação, a situação é ainda mais díspar, pois um aprendiz utilizaria um pré-modificador -ing em apenas $22,62 \%$ das vezes em que um falante nativo o faria com naturalidade.

No item a seguir, são discutidos esses resultados.

\section{DISCUSSÃO DOS RESULTADOS}

A partir do levantamento quantitativo de ocorrências do particípio presente (-ing) como modificador de sintagmas nominais em dois corpora de textos do universo acadêmico, um de aprendizes brasileiros (CorlsF-Inglês) e um de falantes nativos do inglês (LOCNESS), revelou-se uma utilização numericamente mais profícua de -ing modificadores, tanto pré quanto pós, pelos falantes nativos em relação aos aprendizes. Isso pode ser explicado pelo fato de os aprendizes do estudo estarem entre os níveis A2 a B2 de aprendizagem. Nesses níveis, não se espera dos aprendizes a produção de sintagmas nominais muito complexos, envolvendo muitos elementos modificadores. Sobre esse tema, Parkinson e Musgrave (2014) fizeram estudo de corpora e contrastaram a escrita acadêmica de estudantes aprendizes de língua inglesa graduados e mestrandos e constataram que a complexidade dos sintagmas nominais, principalmente em termos de ocorrência de pré e pósmodificadores varia com a proficiência do aprendiz.

Quanto ao uso de pós-modificadores, os falantes nativos empregaram a forma -ing cerca de 2 vezes mais do que os aprendizes brasileiros. O que chama mais a atenção é a diferença no uso do pré-modificador -ing. Neste caso, o déficit na utilização pelos aprendizes é de mais de 4 vezes em relação ao padrão numérico do falante nativo (cf. Gráfico 1). Interpreta-se esse resultado à luz das gramáticas das línguas faladas pelos sujeitos produtores dos textos do CorlsF-Inglês.

Sendo falantes nativos do português, os aprendizes deste estudo têm familiaridade com a pós-modificação do item nominal realizada por um elemento verbal não finito, mais especificamente, por uma construção oracional de particípio presente, na forma -ndo de sua L1, que, grosso modo, corresponde à forma -ing do inglês. Essa familiaridade com a estrutura de L1 possivelmente gerou uma relativa facilidade para a produção de estrutura semelhante na $L 2$, pois houve nos dados uma produção mais numerosa do pós-modificador -ing em relação ao pré-modificador, na proporcionalidade com os -ing produzidos pelo falante nativo (cf. Gráfico 2).

No caso das ocorrências de -ing pré-modificadores, o fato de o português não apresentar a possibilidade de uso do -ndo na posição pré-núcleo do sintagma nominal pode ter contribuído para o número comparativamente bem mais reduzido desse prémodificador nos dados do Corlsf-Inglês (cf. Gráfico 2). Uma possível explicação para as 
ocorrências dessa estrutura, que é alheia ao padrão gramatical da L1 dos aprendizes, pode ser encontrada no processo de aprendizagem pelo qual já passaram. Além disso, foi observada na análise, que a maior parte das ocorrências de pré-modificadores -ing nos textos do Cor-IsF estão em: a) expressões previamente apresentadas nas instruções e/ou nos textos motivadores ou relacionadas à temática proposta ao aprendiz; b) verbos não finitos pré-nucleares que são essencialmente chunks, demonstrando que a produção desses itens reflete expressões frequentemente apresentadas em materiais didáticos e discussões realizadas no contexto das disciplinas de Inglês para Fins Acadêmicos, portanto, estruturas de alguma forma já cristalizadas e c) elementos 'colocados'. Essas situações podem ser vistas a seguir:

a) Título do texto motivador para a tarefa de produção do texto: Young blood to be used in ultimate rejuvenation trial.

Trecho encontrado no corpo do texto motivador: To see if it was linked to the rejuvenating effects, the team gave old mice with enlarged hearts daily injections of GDF11 for 30 days.

Exemplo da influência da temática e das instruções para a tarefa de escrita no Cor-IsF:

(9) Extensive researches have discovered potential rejuvenating effects of young blood plasma transfusions.

b) Exemplo do uso de chunks relacionados ao contexto das disciplinas de Inglês para Fins Acadêmicos:

(10) Indeed, children are generally not worried about making mistakes and tend not to be shy, making the learning process easier.

c) Exemplo do uso de colocados:

(11) So, you need to be carreful about what kind of contend you are posting or sharing in your Facebook, because that can get you in a lot of troubles, meanly in your working life.

Todo esse quadro comparativo pode estar evidenciando um processo de influência da L1 na produção de uma construção específica de L2. Os aprendizes focalizados neste estudo, podem, no geral, estar utilizando proporcionalmente mais -ing pós-modificadores do que pré-modificadores, tornando, dessa forma, mais automatizado o uso do elemento 
não finito na posição pós-núcleo, motivados pela semelhança entre os sistemas linguísticos de L1 e de L2 nessa questão gramatical.

\section{CONCLUSÃO}

Nossa pesquisa investigou, de uma perspectiva quantitativa, o uso de pré e de pósmodificadores do sintagma nominal realizados pelo particípio presente -ing na produção escrita de aprendizes universitários brasileiros de inglês (corpus Corlsf-Inglês) comparativamente à produção escrita de falantes nativos do inglês britânico e estadunidense, também universitários (corpus LOCNESS). Todas as ocorrências estudadas foram selecionadas de linhas de concordância evidenciadas pelo software AntConc a partir de textos de gêneros variados do universo acadêmico. A análise dos dados foi realizada levando-se em conta as similaridades e as diferenças das gramáticas do português e do inglês, referentes à estruturação do sintagma nominal. O particípio presente, em geral, compondo estruturas oracionais (orações adjetivas/relativas reduzidas/não finitas) desempenha a função sintática de pós-modificador de sintagma nominal, tanto na L1 quanto na L2 desses aprendizes. Essa mesma forma não finita do verbo, no entanto, pode ocupar a posição pré-núcleo do sintagma apenas na língua inglesa. Argumentamos, portanto, que a comparação entre os padrões gramaticais das línguas materna e alvo do aprendiz lance luz sobre os resultados deste estudo.

Nesse contexto, em que foi observado o uso que o falante nativo faz do particípio presente como modificador, o aprendiz mostrou uma condição mais propícia à produção de pós do que de pré-modificador com a forma -ing. Já em relação à pré-modificação, pudemos observar que não ocorreu a facilidade de produção dessa estrutura (cf. Quadro 1). No geral, no entanto, o padrão de uso que o aprendiz apresenta relativo ao pré e ao pósmodificador no particípio presente vai na mesma direção do padrão apresentado pelo falante nativo. O que distingue esses dois padrões é a proporção numérica das ocorrências do item gramatical (cf. Gráfico 2).

Reiteramos a importância de um tratamento pedagógico no sentido de se levarem aprendizes brasileiros de inglês a desenvolverem o conhecimento gramatical específico e comparado da estruturação do sintagma nominal em ambas as línguas, a fim de que esses aprendizes avancem em sua interlíngua, passando a ter condição de produzir, de forma mais automatizada, padrões gramaticais da L2. Com Tenuta (1999), advogamos em favor da instrução de aspectos formais da língua-alvo sem desconsiderar o trabalho com a prática do uso da língua estrangeira, ou seja, dos aspectos comunicativos do aprendizado. Nessa perspectiva, a autora salienta que devem ser trabalhadas características do funcionamento da língua-alvo ao mesmo tempo em que se discutiria como o sistema dessa língua não funciona, o que proporcionaria ao aprendiz a condição de checagem de suas hipóteses. Todo esse trabalho pedagógico resultará em ganho de proficiência. O aprendiz, 
nesse processo, vai adquirindo condições de lidar, tanto receptiva quanto produtivamente, com estruturas progressivamente mais complexas, vindo a atingir maior grau de automatização de seu conhecimento da L2.

Por fim, a especificidade da maior facilidade de uso, pelo aprendiz brasileiro, da forma verbal não finita -ing como pós-modificador no SN pode ser vista como um caso de influência da L1 sobre a L2, sendo esse termo interpretado em sentido semelhante ao de motivação. Assim, por conhecimento de um padrão da L1, ocorre mais naturalmente o uso desse mesmo padrão na produção de L2, uma vez que essa situação contribui para o processo de automatização na L2.

Compreendemos o ganho que pode haver em futuras investigações relacionadas ao tema desta pesquisa, no sentido de ampliação de dados, aplicando-se tratamento estatístico a esses dados ampliados, especificando-se os gêneros textuais e/ou separando-se os aprendizes por níveis de proficiência. Além disso, podem ser também realizados estudos na perspectiva experimental, para confirmação de resultados quantitativos desta e das outras possíveis investigações.

\section{AGRADECIMENTOS}

Agradecemos à professora $\mathrm{Dr}^{\mathrm{a}}$ Deise Prina Dutra e sua equipe, da Universidade Federal de Minas Gerais, pela permissão para utilizarmos os dados do CorlsF-Inglês para a realização desta pesquisa.

\section{REFERÊNCIAS}

ALVAREZ, Maria Luisa Ortíz. A transferência, a interferência e a interlíngua no ensino de línguas próximas. In: CONGRESSO BRASILEIRO DE HISPANISTAS, 2, 2002, San Pablo. Associação Brasileira de Hispanistas, Disponível em:<http://www.proceedings.scielo.br/scielo.php?script=sci_arttext\&pid=MSC0000000012002000100039\&lng=en \&nrm $=a b n>$. Acesso em: 14 agosto 2020.

ANTHONY, Laurence. AntConc (Version 3.5.8) [Computer Software]. Tokyo, Japan: Waseda University. 2019. Disponível em https://www.laurenceanthony.net/software. Acesso em: 20 dezembro 2019.

BIBER, Douglas; JOHANSSON, Stig; LEECH, Geoffrey; CONRAD, Susan; FINEGAN, Eduard. Longman Grammar of Spoken and Written English. Harlow: Pearson Education Limited, 1999.

CEGALLA, Domingos Paschoal. Novíssima gramática da língua portuguesa. 48 ed. Rev. São Paulo: Companhia Editorial Nacional, 2008.

CIPRO, Pasquale Neto, INFANTE, Ulisses. Gramática da língua portuguesa. São Paulo: Editora Scipione, 2008.

CORDER, Pete. "A role for the mother tongue”. In: GASS, Susan, SELINKER, Larry. Language transfer in language learning. Amsterdam: John Benjamins Publishing Company, Revised edition, 1992, p. 18-31. 
CUNHA Celso; CINTRA Lindlay. Nova gramática do português contemporâneo. 7. ed., reimpressão. Rio de Janeiro: Lexikon, 2017.

DOWNING, Angela. English grammar. A university course. 3rd. Revised Edition. New York: Routledge, 2015.

DOWNING, Angela; LOCKE, Phillip. A university course in English grammar. Revised Edition. London; New York: Routledge, 2006

DURÃO, Adja B. de A. B. A influência do português como língua materna no processo de aprendizagem de inglês como língua estrangeira: a questão do sujeito gramatical. Revista Investigações, v. 18, n. 2, jul. 2005.

ELLIS, Rod. Understanding Second Language Acquisition. Oxford: Oxford University Press, 1995.

FRICKE, Melinda; ZIRNSTEIN, Megan; NAVARRO-TORRES, Christian; KROLL, Judith. Bilingualism reveals fundamental variation in language processing. Bilingualism: Language and Cognition, vol. 22, no. 1, 2019, p. $200-207$. DOI https://doi.org/10.1017/S1366728918000482 Acesso em: 20 maio 2021.

GASS, Susan M.; SELINKER, Larry. Language transfer in language learning. Amsterdam: John Benjamins Publishing Company, Revised edition 1992

GRANGER, Sylviane. "On identifying the syntactic and discourse features of participle clauses in academic English: native and non-native writers compared”. In:AARTS, Jan; de MÖNNINK, Inge; WEKKER, Herman. Studies in English Language and Teaching. Rodopi: Amsterdam \& Atlanta, 1997, p. 185-198.

HUDDLESTON, Rodney; PULLUM, Geoffrey K. A student's introduction to English grammar. Cambridge: Cambridge University Press, 2005

KROLL, Judith F.; STEWART, Erica. Category interference in translation and picture naming: evidence for asymmetric connections between bilingual memory representations. Journal of Memory and Language, v. 33, p. 149174. DOI:10.1006/imla.1994.1008. Acesso em: 15 setembro 2018.

LANGACKER, Ronald Wayne. Cognitive grammar: An introduction. New York: Oxford University Press, 2008.

MICHAELIS, Dicionário Brasileiro da Língua Portuguesa. São Paulo: Melhoramentos, LTDA, 2015. Disponível em: https://michaelis.uol.com.br/moderno-portugues/. Acesso em: 05 setembro 2021.

OTHERO, Gabriel de Ávila. A gramática da sentença em português: uma descrição formal com um "olho" na implementação computacional. 2008. Tese (Doutorado em Estudos Linguísticos), Faculdade de Letras, Pontifícia Universidade Católica do Rio Grande do Sul, Porto Alegre, 2008.

PARKINSON, Jean; MUSGRAVE, Jill. Development of noun phrase complexity in the writing of English for Academic Purposes students. Journal of English for Academic Purposes. 2014, p. 48-59. DOI:10.1016/i.jeap.2013.12.001. Acesso em: 25 janeiro 2019.

PERINI, Mário Alberto. Gramática descritiva do português. 4 ed. São Paulo: Editora Ática, 2005

RANTA, Elina. Grammar in ELF. In: JENKINS, J. et al. The Routledge Handbook of English as a Lingua Franca. London/New York, Routledge, 2018, p. 244-254.

RUTHERFORD, William E. Second language grammar. Teaching and learning. Harlow: Pearson Education Limited, 1987.

SELINKER, Larry. Interlanguage. International Review of Applied Linguistics, v.10, 1972, p. 209-231. DOI https://doi.org/10.1515/iral.1972.10.1-4.209. Acesso em: 18 maio 2019.

SCHACHTER, Jacquelyn: "A new account of language transfer". In: GASS, Susan, SELINKER, Larry. Language transfer in language learning. Amsterdam: John Benjamins Publishing Company, Revised edition. 1992, p. 32-46.

TENUTA, Adriana Maria. A instrução formal da gramática no ensino/aprendizagem de L2: benefícios e propostas. Revista de Estudos da Linguagem, Belo Horizonte, v.8, n.2, jul./dez. 1999, p. 155-170.

VIÑAS-GUASCH, Nestor; GATHERCOLE, Virginia C. Mueller; STADTHAGEN-GONZALEZ, Hans. Bilingualism and the semantic conceptual interface: the influence of language on categorization. Bilingualism: Language and Cognition, vol. 20, no. 5, 2017, p. 965-979. DOI https://doi.org/10.1017/S1366728916000754. Acesso em: 15 março 2020. 